\title{
Acceptable System Error of Collision Avoidance System Based on the Integrated Error of Driver and System
}

\author{
Keisuke Suzuki ${ }^{1}$ and Makoto Mochizuki ${ }^{2}$ \\ ${ }^{1}$ Kagawa University \\ ${ }^{2}$ Panasonic Corporation, Hayashi-cho, \\ Takamatsu, Kagawa, 761-0396, Japan \\ ksuzuki@eng.kagawa-u.ac.jp
}

\begin{abstract}
This study uses collision-prevention support information at a blind intersection as an example to discuss a methodology for estimating the collision mitigation ratio when the collision avoidance information is used by a driver. It also discusses the acceptable delay time for information presentation regarding a crossing vehicle at a blind intersection. First, driver performance in terms of braking timing and deceleration operation for collision avoidance was analyzed in a driving simulator when the onset timing of the information to notify the driver about a crossing vehicle was changed. Next, a driver model was constructed that simulated a braking operation when a crossing vehicle appeared at a blind intersection where there were no traffic signals. Through Monte Carlo simulations using this driver model, an estimation was made of the frequency of collisions to vehicles crossing the blind intersection. In addition to this estimation, the acceptable delay time for presenting the information was estimated. One of the results indicates that the delay time should be less than $2 \mathrm{~s}$. This means the information should be presented to the driver when the time to collision (TTC) is longer than $3 \mathrm{~s}$ to mitigate the probability of collision.
\end{abstract}

Keywords: Driver Behaviour, Intersection, Face-to-Face Collision, Information for Collision Avoidance, Collision Mitigation, Sensor Reliability, Driver Model.

\section{Motivation of the Study}

This paper proposes a methodology to clarify the acceptable level of the sensors' reliability to reduce collisions at intersections based on the concept of 'integrated error of driver and systems' that authors had reported in previous research [1]-[3]. In that study, an information presentation system was proposed that notified drivers about vehicles crossing blind intersections. This system is used as an example for the discussion and the methodology is used to clarify the acceptable reliability level for the information presentation. This study includes a discussion of this acceptable reliability level, which means the acceptable delay time for presenting the information about crossing vehicles to the driver. First, we investigated the collision-avoidance 
behavior of drivers at traffic intersections, testing 20 drivers in a driving simulator. We constructed a database that captured the driving behavior of the subjects as they decelerated to avoid collisions at the simulated intersection in two different situations. In one situation, the information about the vehicle as it approached the intersection was presented to the driver without any delay time. In the other situation, the information was presented with a delay time. The change in driver behavior when the timing of information presentation was delayed was investigated. Two different timing delays for presenting the information to the driver were used during the experiment. Next, using the database, a driver model that simulated the collision avoidance at the intersection was constructed. The Monte Carlo method was applied for the simulation, and then using this driver model, the collision-avoidance behavior of the drivers was investigated, to estimate not only the effectiveness of the information presentation but also the acceptable delay time of the information presentation. These investigations were carried out, not only during the normal operation of the system but also during delayed operations in which the system presented the information to the driver with delayed timing. Using a methodology that estimates the relationship between the delay time of the information presentation and the effectiveness of the system, the acceptable delay time of the information presentation was clarified quantitatively in this study.

\section{Experimental Method}

\subsection{Experiment Scenario}

Using accident statistical data [4][5] and the results from an investigation using an event data recorder, which analyzed the characteristics of traffic accidents at intersections, we determined the speed of cars travelling on a priority road and that of the cars travelling on a non-priority road. In the visual database of the simulator, we constructed a blind intersection with narrow roads which is typical intersection in the residential area of Japan. We constructed a priority road where the travelling velocity is $40 \mathrm{~km} / \mathrm{h}$, and a non-priority road where the travelling velocity is $20 \mathrm{~km} / \mathrm{h}$. These two roads crossed at an intersection. The width of the road and the position of the obstacles blocking the views from the traffic lanes were specified to match the dimensions of a specific intersection in a metropolitan area of Japan where collisions actually occurred. Each test subject drove a simulated vehicle while it travelled a priority road. A simulation of another vehicle was made to advance into the intersection from the crossing road without deceleration. For each test, the existence of the test subject's vehicle and the direction of this oncoming vehicle advancing into the traffic intersection were set up in a random order. When the time to collision (TTC) became lesser than $1.6 \mathrm{~s}$, the subject driver could visually detect the oncoming vehicle. This condition was specified, based on the database of specific traffic accidents in the metropoli$\tan$ area. In other words, the buildings beside the intersection were placed such that the driver could not view the oncoming vehicle until $1.6 \mathrm{~s}$ before the collision.

When considering the mental condition of the driver when the collision occurred, it is assumed that something was mentally distracting the driver. In this experimental 
study, a mental arithmetic calculation was presented to the drivers while they drove a simulated vehicle, to simulate this mental distraction. Specifically, a numerical figure was presented to the driver every $3 \mathrm{~s}$ and the driver was asked to add this to the previous answer and respond with the first digit as the answer.

\subsection{Collision Avoidance Information}

In the experiments, when the subject's vehicle was approaching the intersection and the crossing vehicle was also approaching the same intersection, the visual and voice information about the vehicle driven by the subject driver was presented to the driver. When the system operated normally without delay time, the notice sound and visual icon displayed on the monitor were presented to the driver $5 \mathrm{~s}$ before the collision (TTC $=5 \mathrm{~s}$ ). The icon notifying the driver about the oncoming vehicle was displayed on a 7-in. monitor installed in the upper part of the instrument panel of the driving simulator at the same time as the notice alarm sounded.

\subsection{Experimental Condition}

Initially, the experiment was set up with a normal condition in which the information was presented to the driver $5 \mathrm{~s}$ before the collision (TTC=5 s). In addition to this normal condition, two other conditions were set up in which the timing of information presentation was delayed for 3 and $4 \mathrm{~s}$, respectively. In these delayed conditions, the information that notified the driver about an oncoming vehicle was presented to the driver when the TTC was 2 and $1 \mathrm{~s}$, respectively. In the condition in which information was presented with a 4-s delay, the driver was presented information only after he/she could visually detect the oncoming vehicle. The subject drivers were not informed that there would be a delay in presenting this information. After the information was presented in normal conditions at TTC $=5 \mathrm{~s}$ to each subject driver nine times, the information was presented once with a delay at the end of the experiment.

\subsection{Subject Drivers}

Twenty men participated in these experiments as the subject drivers. Their average age was 21.8 years and the standard deviation of their ages was 0.7 years. The experimental study in the driving simulator was started after the test subjects gave their informed consent one-week before the experiment and just before the experiment started.

\section{Collision Avoidance Behaviour of the Driver}

\subsection{Braking Behaviour to Avoid Collision during the Simulator Investigation}

In order to construct the database for the simulation that modeled the braking behavior of drivers in time series simulation, the subject drivers' behavior was 
investigated. The accumulated frequency distribution for each analysis index that explains the driver's braking operation is shown in Figure 1.

First, we focus on the braking reaction time. The initiation timing of the subject drivers' braking operation was delayed when there was a delay in presenting the information to the driver, compared with the condition when the system operated normally. When the information was presented to the driver without delay at the timing of TTC $=5 \mathrm{~s}$, the driver started braking at the earliest time. Therefore, when the information was presented to the driver at the timing of TTC $=5 \mathrm{~s}$, the maximum deceleration and average jerk were smaller. The braking reaction time to avoid the collision after the information presentation was extremely short in a condition with a 4-s delay. In the experimental condition of a 4-s delay, information was presented to the driver $0.6 \mathrm{~s}$ after the moment when the driver could view the vehicle at the traffic intersection. When the information was presented to the driver at TTC $=1 \mathrm{~s}$ in the experimental condition of a 4-s delay, the subject driver was able to visually detect the crossing vehicle before the information was presented to the driver. Thus, the driver started braking to avoid a collision with the crossing vehicle before the information was presented to the driver.

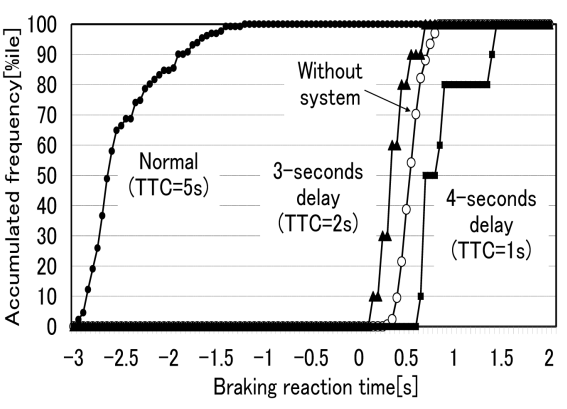

(a) Braking reaction time

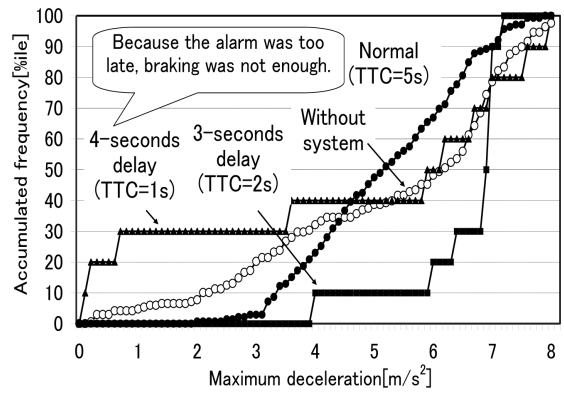

(b) Maximum deceleration during braking

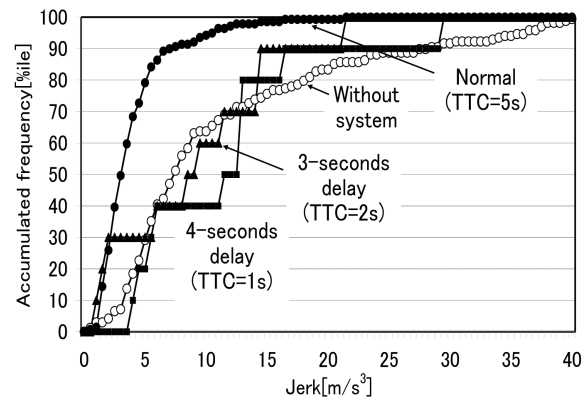

(c)Average jerk during deceleration

Fig. 1. Drivers' braking behaviour to avoid collision with a crossing vehicle at a blind intersection 
The database which quantifies the characteristics of drivers' braking behavior was inputted into the driver model that was constructed by the authors in this study, and the collision avoidance behavior of the drivers was simulated by applying a Monte Carlo simulation method which could conduct the time-series simulation of the braking operation of the drivers. This simulation analysis demonstrated the collision frequency quantitatively in each condition. Based on the results of mitigation level of traffic accidents, we were able to clarify the delay time of information presentation that is acceptable in reducing accidents.

\subsection{Simulation of the Braking Behaviour of Drivers at Traffic Intersections}

While estimating the effect of utilizing the collision avoidance system to reduce accidents, a higher level of accuracy of the estimation should be maintained. For this reason, it is necessary to conduct the experimental study as many times as possible so that a statistical confidence interval; i.e., the error of estimation, becomes as small as possible. In this research, the driver model for simulating the braking behavior at the blind intersection was constructed in order to quantify the collision mitigation ratio during the use of information presenting systems used for collision avoidance. By using this driver model, we simulated the behavior of 10,000 avoidances of collisions in critical situations. The variance of drivers' behavior for each delay time for presenting information by applying the Monte Carlo simulation was investigated. In a simulation study, if the statistical confidence interval is small enough and the statistical reliability is maintained high enough, the collision mitigation ratio is quantified. By inputting the driver data concerning braking reaction time, maximum deceleration and averaged jerk in the simulation model, the change of collision-avoidance behavior of drivers and the effectiveness of information presentation were investigated through Monte Carlo simulation.

\subsection{Quantifying the Collision Frequency}

By using the driver model, the collision frequency was quantitatively investigated for each experimental condition with different levels of delay time for presenting information. The investigation results concerning the collision frequency and collision velocity, which we investigated through a Monte Carlo simulation by using the driver model, are shown in Table 1.

When the system operated normally without a delay time for presenting information (TTC $=5 \mathrm{~s}$ ), the collision at the traffic intersection did not occur. By comparing the collision frequency when the system was used with the collision frequency when the system was not used, the drivers' ability to mitigate the collision at the traffic intersection is clearly shown. When the driver became used to the normal information timing (TTC $=5 \mathrm{~s}$ ) and the information presentation was performed at a delayed timing such as $3 \mathrm{~s}(\mathrm{TTC}=2 \mathrm{~s})$ or $4 \mathrm{~s}(\mathrm{TTC}=1 \mathrm{~s})$, the collision frequency became extremely high. 
Table 1. The probability of collision within each operating situation of the information system

\begin{tabular}{c|c}
\hline \hline Without system & $7.23 \times 10-1 \quad(72.3 \%)$ \\
\hline Normal & 0.00 \\
\hline $\begin{array}{c}\text { 2-seconds delay } \\
(\mathrm{TTC}=3 \mathrm{~s})\end{array}$ & $\begin{array}{c}0.00 \\
\text { *estimated by use of the data in case of 3-seconds } \\
\text { delay }\end{array}$ \\
\hline $\begin{array}{c}\text { 3-sedonds delay } \\
\text { (TTC=2s) }\end{array}$ & $8.19 \times 10-1 \quad(81.9 \%)$ \\
\hline $\begin{array}{c}\text { 4-sedonds delay } \\
(\mathrm{TTC}=1 \mathrm{~s})\end{array}$ & $1.00 \quad(100 \%)$ \\
\hline
\end{tabular}

\section{Acceptable Level of Sensor Reliability Based on the Integrated Error of the Driver-System}

\subsection{Definition of the Integrated Error of the Driver-System}

By substituting the value of collision frequency, shown in Table 1, into the integrated error of the driver-system [1]-[3], it is possible to estimate the total error for the manmachine system when the driver used the information. By comparing the collision frequency when the information was not used with that when the information was used but delayed, we quantitatively estimated the acceptable level of the delay time for presenting information. The details are as follows.

First, in Figure 2, the depiction of the integrated error of the driver-system that authors proposed in a previous research [1]-[3] is shown. We used this to analyze the accident reduction ratio of the driving assistance system. The probability of driver error is plotted on the abscissa, and the probability of a system error is plotted on the ordinate. The integrated error probability as a man-machine system can be calculated by multiplying the error probability of the driver by the error probability of the system. Each parameter is defined below.

- Ed0: Driver operation error probability when the system is not used.

- Ed1: Driver operation error probability when the system is used and the information is delayed.

- Ed2: Driver operation error probability when the system is used and the information is not delayed but the driver does not suitably evade the accident.

- Es: System error probability

The sum total of the area of the domains indicated by \#1 and \#2 represents an integrated error when a driver uses the information system. The area of the domain enclosed with a thick broken line $(\operatorname{Ed} 0 \times 1)$ indicates the error probability when a driver does not use the information system. A driving-support system that presents information effectively reduces accidents if the area of the grey domain $(\# 1+\# 2)$ becomes sufficiently smaller than the area of the domain enclosed within the dashed line. 


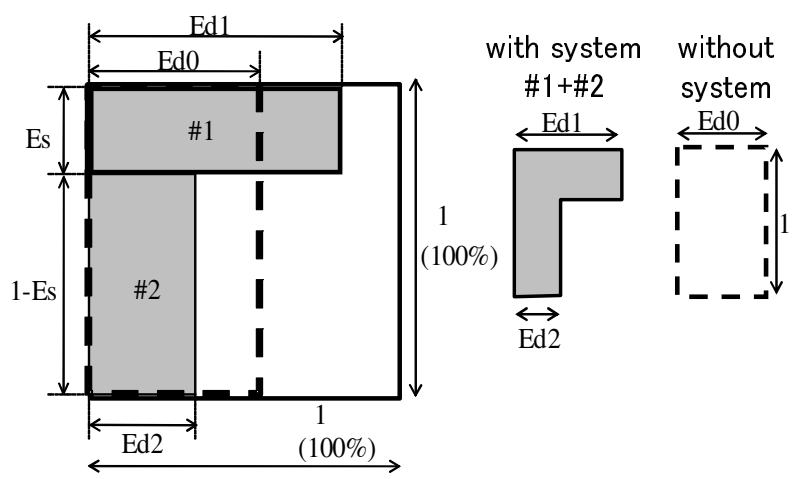

Fig. 2. Analysis model for the integrated error of driver-system

The optimal value of the mitigation ratio of collision ((“\#1 + \#2") / ("Ed0 $\times 1$ ")) is an important factor in this study. The smaller risk is better to minimize traffic accidents. However, to achieve such low risk levels, it becomes more difficult to achieve the necessary high levels of reliability of the sensors. In this study, the optimal value for the mitigation ratio of collision was set at 1/100 based on the authors' previous study regarding the const-benefit analysis.

\subsection{Acceptable Level of Delay Time for Information Presentation}

Based on the above model, the effectiveness of information presentation and an acceptable delay time for the information presentation are discussed. The simulation result in terms of collision frequency was investigated using the above-mentioned driver model, and the numerical values in Figure 2 were determined as follows. In this study, the optimal value of the collision mitigation ratio was set at $1 / 100$. This means that the integrated error probability shown by the total areas of \#1+\#2 should be 1/100 of Ed0. With respect to this requirement, the possible Es can be estimated quantitatively. In other words, the relationship between the delay time for presenting the information and the acceptable probability in terms of this delay time are shown in Figure 3. In Figure 3, the acceptable probability in each delay time is shown. For instance, the acceptable time frequency should be less than $0.883 \%$ in a condition in which the information is delayed for $3 \mathrm{~s}$. This means that if the information presentation is delayed for $3 \mathrm{~s}$ and information is presented at TTC $=2 \mathrm{~s}$, the collision frequency will be $1 / 100$ of that when the system is not used. It was also clarified that if the information presentation is delayed for less than $2 \mathrm{~s}$ and information is presented before TTC $=3 \mathrm{~s}$, the driver can avoid the collision. Therefore, it can be concluded that the delay time for presenting the information should be shorter than $2 \mathrm{~s}$ in order to present the information earlier than the timing of TTC $=3 \mathrm{~s}$. 


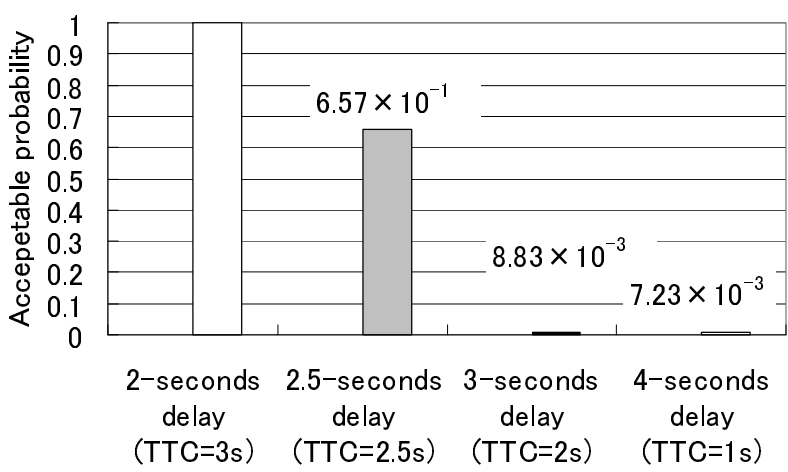

Fig. 3. Acceptable level meaning the acceptable delay time for the information presentation

\section{Summary}

In the experiments using a driving simulator, a database concerning the braking actions taken to avoid collisions at a blind intersection was constructed. Next, a driver model was constructed that could reproduce these collision evasive actions. By applying the Monte Carlo simulation method for this driver model, and considering the variance of driver behavior, we were able to quantitatively show the accident reduction effect when using the system. The frequency of collision and collision velocity estimated through the simulation for each condition is shown as follows.

- The frequency of collision

- Without information: $7.23 \times 10-1,72.3 \%$

- Normal operation (Information was presented at TTC=5s): 0.00

- Delayed operation "3-seconds delay" (Information was presented at TTC=2s): $8.19 \times 10-1,81.9 \%$

- Delayed operation "4-seconds delay" (Information was presented at TTC=1s): $1.00,100 \%$

The acceptable delay time for presenting the information can be estimated as follows. This analysis is based on the idea that the information system should be put into the market for practical use when the frequency of collision is less than $1 / 100$. This frequency of collision (the total risk when the driver used the system) was estimated based on the total risk of the man-machine system, which was proposed by authors and named the 'integrated error of the driver-system'. It can be concluded that the delay time for presenting the information should be shorter than $2 \mathrm{~s}$ in order to present the information earlier than the timing of TTC $=3 \mathrm{~s}$. 
- Acceptable time frequency for each delay time

- Delayed operation "3-seconds delay" (Information was presented at TTC $=2 \mathrm{~s}$ ) less than $8.83 \times 10-3(0.883 \%)$

- Delayed operation "4-seconds delay" (Information was presented at TTC=1s) less than $7.23 \times 10-3(0.723 \%)$

\section{References}

1. Suzuki, K., Yamada, K.: Method for Evaluating Effectiveness of Information Presentation in Terms of Collision Avoidance. International Journal of Intelligent Transportation Systems Research 9(1), 37-46 (2011), doi:10.1007/s13177-010-0023-8

2. Suzuki, K., Tanaka, H., Miichi, Y., Aga, M.: Methodology for Quantifying DamageMitigation Level of Collision-Avoidance Braking System. In: Proceedings of First International Symposium on Future Active Safety Technology-FAST-ZERO, TS2-8-3-3 (2011)

3. Suzuki, K., Yamada, K.: Method for evaluating the collision mitigation ratio when using collision avoidance alarm at intersection. In: Proceedings of SICE Annual Conference, \#TC18-06 (2010)

4. Institute for Traffic Accident Research and Data Analysis, Traffic accident investigation and an analysis report (2009)

5. http://www8.cao.go.jp/koutu/chou-ken/h19/houkoku.pdf 